\title{
Long-term mortality after intracerebral hemorrhage
}

\author{
M.L. Flaherty, MD; M. Haverbusch, BSN; P. Sekar, MS; B. Kissela, MD; D. Kleindorfer, MD; \\ C.J. Moomaw, PhD; L. Sauerbeck, RN, MS; A. Schneider, MD; J.P. Broderick, MD; and D. Woo, MD
}

\begin{abstract}
Objective: To characterize long-term mortality following intracerebral hemorrhage (ICH) in two large population-based cohorts assembled more than a decade apart. Methods: All patients age $\geq 18$ hospitalized with nontraumatic ICH in the Greater Cincinnati/Northern Kentucky area were identified during 1988 (Cohort 1) and from May 1998 to July 2001 and August 2002 to April 2003 (Cohort 2). Mortality was tabulated using actuarial methods and compared with a log-rank test. Results: There were 183 patients with ICH in Cohort 1 and 1,041 patients in Cohort 2. Patients in Cohort 1 were more likely to be white $(p=0.024)$ and undergo operation for their ICH $(p=0.002)$, whereas patients in Cohort 2 were more commonly on anticoagulants $(p<0.001)$. Among patients in Cohort 1 , mortality at 7 days, 1 year, and 10 years was 31,59 , and $82 \%$. Among patients in Cohort 2, mortality at 7 days and 1 year was 34 and $53 \%$. Mortality rates did not differ between cohorts by log-rank test $(p=0.259)$. Conclusions: Intracerebral hemorrhage (ICH) mortality did not improve significantly between study periods. Operation for ICH became less frequent, whereas anticoagulant-associated ICH became more common.
\end{abstract}

NEUROLOGY 2006;66:1182-1186

Recent investigations into the surgical and medical management of intracerebral hemorrhage (ICH) prove that large-scale clinical trials for this condition are feasible and provide hope that new treatments may improve patient outcomes. ${ }^{1,2}$ Outside of clinical trials, changes in medical practice and the natural history of disease are ideally documented in population-based studies that capture all disease cases within a defined community. To date, most population-based studies of ICH outcome have been small and racially homogeneous. ${ }^{3}$ Limited sample size has precluded analysis of some predictors of ICH outcome, such as location of hemorrhage and anticoagulant use in most of these studies. ${ }^{3,4}$ Furthermore, whereas short-term mortality following ICH is known to be high, the pattern of long-term mortality following ICH and recent trends in ICH mortality have not been well documented. ${ }^{3}$ We present a population-based study of ICH outcome, including long-term mortality data, stratification by location of hemorrhage, and a comparison of two large ICH cohorts assembled more than a decade apart.

Methods. Two ICH cohorts from the five-county Greater Cincinnati/Northern Kentucky area (GCNK) were tracked for this study. Cohort 1 was assembled from January 1988 through December 1988 and has been the subject of previous reports. ${ }^{5,6}$ Cohort 2 was assembled from May 1998 through July 2001 and August 2002 through April 2003 as part of the Genetic and Environmental Risk
Factors for Hemorrhagic Stroke (GERFHS) study, an ongoing population-based study of ICH and subarachnoid hemorrhage in the GCNK region. ${ }^{7}$ The methodology of the GERFHS study has been previously described. 7,8

The current study includes all hospitalized cases of ICH that occurred in persons age $\geq 18$ within the five GCNK metropolitan counties during the prescribed periods. For both cohorts, cases were identified by retrospective review of primary and secondary International Classification of Diseases-9 (ICD-9) codes. For Cohort 1, ICD-9 codes 430, 431, 432.9, 436, 437.3, and 747.81 were utilized. ${ }^{6}$ For Cohort 2, ICD-9 codes 430 through 432 were utilized through October 1999 and codes 430 through 438.9 thereafter. For Cohort 2, study nurses also maintained active surveillance ("hot pursuit") at several hospitals that treat most ICH and subarachnoid hemorrhage in the area by reviewing neurosurgery logs and patient rosters several times each week. ${ }^{7}$ In both periods, potential cases were abstracted by study nurses and reviewed in detail by study physicians. Residents of the five-county GCNK region seek care almost exclusively at 1 of the 16 participating metropolitan hospitals. ${ }^{6}$ Patients living within the 50-mile radius required by the GERFHS study but outside of the five counties of interest were excluded by zip code of residence. For the current study, exclusion criteria applied to both cohorts were previous ICH, traumatic ICH, hemorrhagic cerebral infarction, and hemorrhage associated with brain tumor, encephalitis, endarterectomy, and thrombolytic treatment of ischemic stroke. ICHs associated with vascular malformations or anticoagulation were included.

Patient demographics and putative risk factors for ICH were recorded for each cohort by chart review and compared with the $\chi^{2}$ test, Student $t$ test, or Wilcoxon two-sample test as appropriate. For any case in which ICH location was not unequivocally identified by radiographic reports, radiographic films were reviewed by investigators. Surgical intervention for ICH was defined as hemicraniectomy, craniotomy for removal of a hematoma or vascular malformation, or stereotactic drainage of a hematoma. CSF drain-

From the Departments of Neurology (M.L.F., M.H., B.K., D.K., C.J.M., L.S., A.S., J.P.B., D.W.) and Environmental Health (P.S.), University of Cincinnati Medical Center, OH.

Dr. Schneider's current address is Mission Hospitals, Asheville, NC.

Supported in part by the National Institute of Neurological Disorders and Stroke (R-01-NS 36695).

Disclosure: The authors report no conflicts of interest.

Received June 27, 2005. Accepted in final form January 18, 2006.

Address correspondence and reprint requests to Dr. M.L. Flaherty, University of Cincinnati Medical Center, 231 Albert Sabin Way, MSB Rm. 5161B, Cincinnati, OH, 45267-0525; e-mail: matthew.flaherty@uc.edu

1182 Copyright (c) 2006 by AAN Enterprises, Inc. 
Table 1 Comparison of ICH patient demographics, 1988 vs 1998-2003

\begin{tabular}{lccc}
\hline & 1988 & $1998-2003$ & $p$ Value \\
\hline Total cases & 183 & 1,041 & \\
Age, mean (SD); y & $70.3(15.0)$ & $70.0(15.3)$ & 0.798 \\
Female (\%) & $104(56.8)$ & $576(55.2)$ & 0.689 \\
White (\%) & $157(85.8)$ & $817(78.5)$ & 0.024 \\
Location of ICH (\%) & & & 0.605 \\
$\quad$ Deep cerebral & $88(48)$ & $516(50)$ & \\
$\quad$ Lobar & $71(39)$ & $358(34)$ & \\
$\quad$ Cerebellar & $14(8)$ & $102(10)$ & \\
$\quad$ Brainstem & $10(6)$ & $65(6)$ & \\
Admit GCS, mean* & 11.0 & 10.9 & 0.714 \\
Admit GCS, median* & 12.0 & 14.0 & 0.004 \\
Hypertensive (\%) & $126(68.9)$ & $693(66.6)$ & 0.545 \\
Diabetic (\%) & $35(19.1)$ & $202(19.4)$ & 0.930 \\
Anticoagulation $\dagger(\%)$ & $9(4.9)$ & $190(18.3)$ & $<0.001$ \\
Operation for ICH (\%) & $33(18.0)$ & $104(10.0)$ & 0.002 \\
Intraventricular drain $\%(\%)$ & $24(13.1)$ & $96(9.2)$ & 0.102 \\
\hline
\end{tabular}

* For 1988 , values are available for $182 / 183$ patients. For $1998-$ 2003 , values are available for 910/1,041 patients.

$\dagger$ Anticoagulation $=$ warfarin, heparin, or low molecular weight heparin.

† Data available for all cases in 1988 and 1,017/1,041 cases in 1998-2003

$\mathrm{ICH}=$ intracerebral hemorrhage; GCS = Glasgow Coma Scale.

age was recorded separately. Hematoma volumes and the presence of intraventricular hemorrhage were not routinely recorded. Survival after ICH was calculated using actuarial methods after querying the 1988 study database, GERFHS study records, the Social Security Death Index, and Ohio and Kentucky death registers. Persons not documented to be deceased were assumed to be alive. Survival curves were compared by log-rank test. To determine if era of hemorrhage (i.e., membership in Cohort 1 or Cohort 2) was an independent predictor of survival after ICH, a Cox regression model for survival among all subjects was created using the variables from table 1 plus era of hemorrhage. Variables with a $p$ value of $>0.10$ were backward eliminated from the model, with era of hemorrhage forced into the final model. Because admission Glasgow Coma Scale (GCS) scores were found to be highly dependent upon hemorrhage location, the model was run with and without GCS score as a variable. The model with GCS score as a variable excluded one patient from Cohort 1 and 131 patients from Cohort 2 because of missing GCS scores. The institutional review board for each participating hospital system approved the GERFHS study.

Results. Cohort 1 included 183 persons. Cohort 2 included 1,041 persons after exclusion of 3 cases because of insufficient information. Patient demographics and putative $\mathrm{ICH}$ risk factors are compared by era in table 1 . Patients in Cohort 1 were more likely to be white $(p=0.024)$ and undergo operation for their ICH $(p=0.002)$, whereas patients in Cohort 2 were more commonly on anticoagulants $(p<0.001)$, with a greater than threefold increase in the percentage of anticoagulant-associated hemorrhages in the latter group. Mean GCS scores were similar between cohorts, but the median GCS score was higher in Cohort 2 (12 vs $14 ; p=0.004)$. The balance of hemorrhage locations

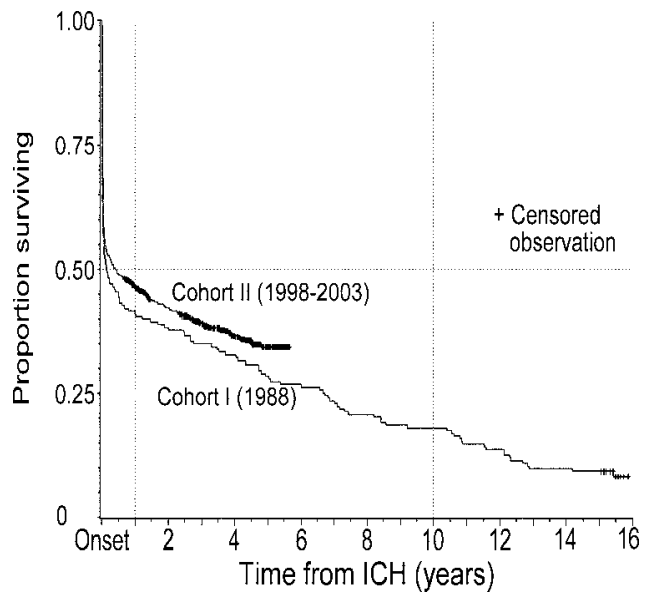

Figure 1. Comparison of intracerebral hemorrhage (ICH) mortality. Cohort 1 = 1988; Cohort $2=1998-2003$.

was similar between groups $(p=0.605)$. A survival curve comparing cohorts is presented in figure 1. Among patients in Cohort 1, mortality at 7 days, 1 month, 1 year, and 10 years was $31,48,59$, and $82 \%$. Among patients in Cohort 2 , mortality at 7 days, 1 month, and 1 year was 34, 44, and 53. Mortality did not differ between cohorts by log-rank test $(p=0.259)$. Survival stratified by ICH location for all patients is presented in figure 2. Thirty-day and 1-year mortality rates were 44 and 52\% for deep cerebral ICH, 46 and $58 \%$ for lobar ICH, 60 and $68 \%$ for brainstem ICH, and 34 and $45 \%$ for cerebellar ICH. Mortality differed by location by log-rank test $(p=0.022)$.

Results reaching a significance level of $<0.10$ in the Cox regression model of survival among all patients (excluding GCS as a variable) are presented in table 2. Predictors of mortality in this model were increasing age, diabetes, anticoagulation, and brainstem location of hemorrhage. Cerebellar location of ICH and operation for ICH were associated with better survival. There were trends toward better survival for patients from Cohort 2 as compared with Cohort 1 ( $p=0.13)$, for deep cerebral location as compared with lobar location ( $p=0.11$ ), and for African Americans as compared with whites $(p=0.101)$. The relationship between GCS and survival was found to be quadratic rather than linear, and so in the Cox model with GCS, both quadratic and linear terms for GCS were included. In

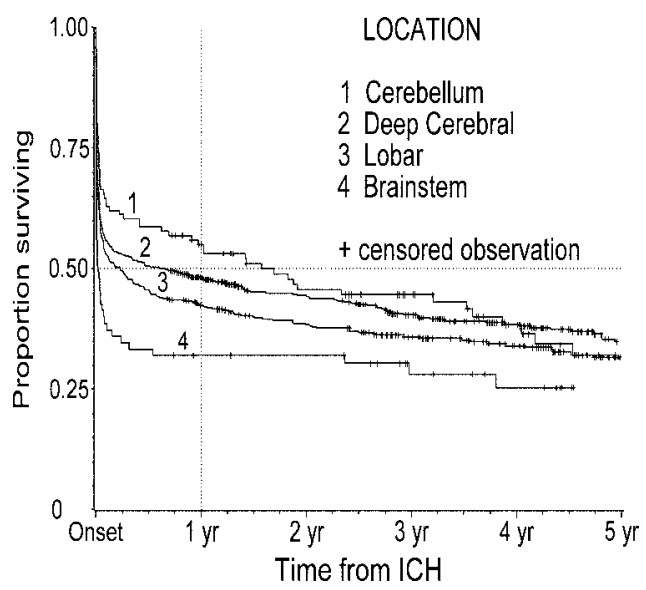

Figure 2. Survival after intracerebral hemorrhage (ICH) stratified by location. 
Table 2 Cox regression model of ICH outcome

\begin{tabular}{lccr}
\hline & $\begin{array}{c}\text { Hazard } \\
\text { ratio* }\end{array}$ & $95 \%$ CI & $p$ Value \\
\hline $\begin{array}{l}\text { Location of ICH } \dagger \\
\quad \text { Cerebellar }\end{array}$ & 0.75 & $0.58-0.98$ & 0.033 \\
$\quad$ Deep cerebral & 0.88 & $0.76-1.03$ & 0.106 \\
$\quad$ Brainstem & 1.37 & $1.02-1.82$ & 0.036 \\
Operation for ICH & 0.74 & $0.58-0.96$ & 0.022 \\
Period of ICH (Cohort 2 vs 1) & 0.86 & $0.71-1.05$ & 0.133 \\
Age $\neq$ & 1.03 & $1.02-1.03$ & $<0.001$ \\
Diabetes & 1.25 & $1.06-1.49$ & 0.010 \\
Anticoagulation & 1.49 & $1.24-1.79$ & $<0.001$ \\
\hline
\end{tabular}

* Hazard ratios of $>1$ indicate increased risk for death.

$\uparrow$ Lobar ICH used as referent.

$\ddagger$ For each increasing year of age.

$\mathrm{ICH}=$ intracerebral hemorrhage.

this model, anticoagulation, increasing age, and diabetes remained predictors of mortality, whereas operation for ICH remained protective. Increasing GCS score was associated with better outcomes $(p=0.005)$, whereas location of ICH became nonsignificant. Era of hemorrhage remained nonsignificant.

Discussion. Our study confirms the high mortality rate following ICH reported in smaller populationbased studies. The 1-month case fatality rates of $48 \%$ (Cohort 1) and 44\% (Cohort 2) are comparable with the $42 \%$ rate estimated from other population-based studies. $^{3}$ Patients with ICH fare worse than those with ischemic stroke, and few are left without disability. ${ }^{3,5}$ After a sharp drop in survival immediately following ICH, a more gradual decline resulted in 10 -year survival of only $18 \%$ in Cohort 1.

Mortality following ICH did not differ significantly between our cohorts, which were separated by more than a decade. This reflects the lack of proven effective treatments for this condition. Mortality after ICH was reportedly as high as $90 \%$ in the pre-CT area. ${ }^{9}$ The lower mortality in our cohorts was likely due to a combination of identification bias in the pre-CT era (with mild hemorrhages misclassified as ischemic infarcts) and improved supportive care. ${ }^{10}$ Continuing refinements in neuroimaging and neurointensive care did not translate into a substantial reduction in mortality between 1988 and 1998 through 2003 in our area.

The most striking difference in our cohorts was the variation in risk factor profiles. The groups were similar in patient age and sex as well as the prevalence of hypertension and diabetes. Cohort 1 was more likely to be white, partly reflecting the changing racial balance in our five-county area $(84.5 \%$ white in 1990 vs $82.4 \%$ white in 2000 ). The median admission GCS score was higher in Cohort 2 than Cohort 1. Although missing data prohibit a definitive conclusion about the cause of this change, it is possi- ble that more frequent and improved neuroimaging resulted in the proper classification of smaller hemorrhages. A second possibility is that patients in Cohort 2 arrived in the emergency department more quickly than patients in Cohort 1 and had their GCS scores documented before the delayed deterioration sometimes seen with hematoma expansion. Our data were insufficient to allow a detailed comparison of arrival times by period. Another notable difference between cohorts was a $13 \%$ absolute increase in the percentage of anticoagulant-associated hemorrhages from 1988 to 1998 through 2003. This increase occurred after the publication of trials demonstrating the efficacy of warfarin for the primary and secondary prevention of ischemic stroke among patients with atrial fibrillation, and it coincides with data showing increased warfarin use in these populations. ${ }^{11-14}$ Patients with anticoagulant-associated $\mathrm{ICH}$ had poorer outcomes in our Cox regression model, consistent with previous reports demonstrating greater risk of hematoma expansion and worse survival among anticoagulated ICH patients than those without coagulopathy. ${ }^{15,16}$ Increasing age and diabetes have likewise been associated with worse outcomes. ${ }^{17-19}$ The use of surgery for ICH diminished in our population between 1988 and 2003, in concert with a growing number of negative surgical treatment trials. ${ }^{20,21}$ Whereas surgery showed a protective effect in the Cox model, this may be explained by a selection bias not captured by the available data.

Location of ICH had an effect upon survival that was not adequately described by dichotomizing hemorrhages as supratentorial vs infratentorial or lobar vs deep. Brainstem ICH proved the most lethal subtype, whereas cerebellar ICH had the best prognosis. In these large cohorts, lobar ICH and deep cerebral ICH had similar case fatality rates, contrary to some older reports describing lobar ICH as more often benign. ${ }^{22,23}$ Other studies have shown that lobar hemorrhages have greater average volume than deep cerebral hemorrhages but are less likely to extend into the ventricular system, two counterbalancing influences on prognosis. ${ }^{4,24}$

The largest previous population-based study of ICH outcome was performed in Izumo City, Japan. ${ }^{4}$ The authors reported on 350 patients ascertained from 1991 to 1998 , excluding patients with vascular malformations or coagulopathy. Outcomes in their cohort were remarkably good, with 7 - and 30-day mortality rates of 11 and $13 \%$. As in our study, in Izumo City, survival was best after cerebellar ICH and worst after brainstem ICH. Thirty-day case fatality rates in their population were $0 \%$ for cerebellar ICH, $11 \%$ for lobar ICH, 9 to $14 \%$ for different deep cerebral locations, and 53\% for brainstem ICH. In a Cox regression analysis of 3-month outcome in their cohort, ICH location was the strongest independent predictor of survival, followed by hematoma volume and admission GCS score. The authors suggest that their low mortality rates may be due to aggres- 
sive management (including surgery) and early treatment of patients at hospitals with neurosurgical support. These explanations seem unlikely given the lack of benefit in surgical ICH trials and the fact that most ICH patients in our area are transferred immediately to a tertiary center..$^{1,20}$ Although it is possible that patients with severe hemorrhages have care withdrawn more frequently in Cincinnati than Izumo City, only $30 \%$ of survivors in Izumo City were left in a vegetative state or with severe disability. This figure is lower than described in western reports and would not be expected if neurologically devastated patients had life sustained by aggressive, prolonged intervention. ${ }^{5}$

Other possible reasons for the different outcomes were the Japanese exclusion of anticoagulantassociated hemorrhages and differences in admission GCS scores. ${ }^{15}$ In Izumo City, $16 \%$ of patients presented with a GCS score of 3 to 6 compared with $27 \%$ of patients in Cincinnati. Admission GCS score is presumably a reflection of the disease state and the patient's neurologic reserve and is not itself a causal force. In our series, GCS score was highly dependent upon ICH location. When GCS was included in our Cox model, location of ICH became nonsignificant. This may have occurred because we were unable to account for hematoma size and intraventricular hemorrhage, two important factors that may influence GCS score. Because we did not record these values, we cannot determine whether they differed between the populations of Cincinnati and Izumo City.

This study was limited by our inability to control for some of the known variables (hematoma volume, intraventricular hemorrhage) and unknown variables that influence outcome after $\mathrm{ICH} .{ }^{19}$ Our goal in this setting was to describe survival following ICH in a population-based setting, given existing practice patterns in our large metropolitan area, rather than to develop a comprehensive model or algorithm for survival after ICH as has been previously done. ${ }^{19,25}$

The fact that survival in our ICH population has not improved significantly in the last 10 to 15 years suggests that changes in practice during that time did not have a major clinical impact. It is possible that improvements in patient care have been counterbalanced by other negative prognostic factors such as the increase in anticoagulant-associated ICH. Alternatively, positive prognostic factors such as smaller hemorrhages detected by more frequent imaging may have improved survival in Cohort 2 (and produced higher median admission GCS scores). The problem of the "self-fulfilling prophecy" in patients with neurologic catastrophes is well recognized (i.e., the perception that survival is impossible, followed by withdrawal of care and death of the patient). ${ }^{26}$ Patterns of care following ICH may have changed between study periods, with more patients in the current era having care withdrawn. We did not record the prevalence of do-not-resuscitate status or orders for comfort care, and so we cannot account for this possibility.

This study provides a comprehensive view of longterm mortality following ICH and emphasizes the fundamental challenge of ICH treatment: The majority of ICH deaths occur very shortly after disease onset. Any meaningful reduction in the medical and social burden caused by ICH must therefore arise from primary prevention, very early intervention after hemorrhage, or novel rehabilitation strategies. The survival curves presented will serve as useful baselines for comparison as new therapies for ICH become available.

\section{Acknowledgment}

The authors thank Prof. Richard Hornung for statistical advice.

\section{References}

1. Mendelow AD, Gregson BA, Fernandes HM, et al., for the STICH investigators. Early surgery versus initial conservative treatment in patients with spontaneous supratentorial intracerebral haematomas in the International Surgical Trial in Intracerebral Haemorrhage (STICH): a randomised trial. Lancet 2005;365:387-397.

2. Mayer SA, Brun NC, Begtrup K, et al., for the Recombinant Activated Factor VII Intracerebral Hemorrhage Trial Investigators. Recombinant activated factor VII for acute intracerebral hemorrhage. N Engl J Med 2005;352:777-785.

3. Dennis MS. Outcome after brain haemorrhage. Cerebrovasc Dis 2003;16 (suppl 1):9-13.

4. Inagawa T, Ohbayashi N, Takechi A, Shibukawa M, Yahara K. Primary intracerebral hemorrhage in Izumo City, Japan: incidence rates and outcome in relation to the site of hemorrhage. Neurosurgery 2003;53: 1283-1298.

5. Broderick J, Brott T, Tomsick T, Tew J, Duldner J, Huster G. Management of intracerebral hemorrhage in a large metropolitan population. Neurosurgery 1994;34:882-887.

6. Broderick JP, Brott T, Tomsick T, Huster G, Miller R. The risk of subarachnoid and intracerebral hemorrhages in blacks as compared with whites. N Engl J Med 1992;326:733-736.

7. Flaherty ML, Woo D, Haverbusch M, et al. Racial variations in location and risk of intracerebral hemorrhage. Stroke 2005;36:934-937.

8. Woo D, Sauerbeck LR, Kissela BM, et al. Genetic and environmental risk factors for intracerebral hemorrhage: preliminary results of a population-based study. Stroke 2002;33:1190-1196.

9. Broderick JP, Phillips SJ, Whisnant JP O'Fallon WM, Bergstralh EJ. Incidence rates of stroke in the eighties: the end of the decline in stroke? Stroke 1989;20:577-582.

10. Drury I, Whisnant JP, Garraway WM. Primary intracerebral hemorrhage: impact of CT on incidence. Neurology 1984;34:653-657.

11. Hart RG, Halperin JL, Pearce LA, et al. Lessons from the stroke prevention in atrial fibrillation trials. Ann Intern Med 2003;138:831-838.

12. European Atrial Fibrillation Trial Study Group. Secondary prevention in non-rheumatic atrial fibrillation after transient ischemic attack or minor stroke. Lancet 1993;342:1255-1262.

13. Fang MC, Stafford RS, Ruskin JN, Singer DE. National trends in antiarrhythmic and antithrombotic medication use in atrial fibrillation. Arch Intern Med 2004;164:55-60.

14. Osseby GV, Benatru I, Sochurkova D, et al. Trends in utilization of antithrombotic therapy in patients with atrial fibrillation before stroke onset in a community-based study, from 1985 through 1997. From scientific evidence to practice. Prev Med 2004;38:121-128.

15. Rosand J, Eckman MH, Knudsen KA, Singer DE, Greenberg SM. The effect of warfarin and intensity of anticoagulation on outcome of intracerebral hemorrhage. Arch Intern Med 2004;164:880-884.

16. Flibotte JJ, Hagan N, O’Donnell J, Greenberg SM, Rosand J. Warfarin, hematoma expansion, and outcome of intracerebral hemorrhage. Neurology 2004;63:1059-1064.

17. Arboix A, Massons J, Garcia-Eroles L, Oliveres M, Targa C. Diabetes is an independent risk factor for in-hospital mortality from acute spontaneous intracerebral hemorrhage. Diabetes Care 2000;23:1527-1532.

18. Passero S, Ciacci G, Ulivelli M. The influence of diabetes and hyperglycemia on clinical course after intracerebral hemorrhage. Neurology 2003;61:1351-1356.

19. Hemphill JC, 3rd, Bonovich DC, Besmertis L, Manley GT, Johnston SC. The ICH score: a simple, reliable grading scale for intracerebral hemorrhage. Stroke 2001;32:891-897.

20. Hankey G, Hon C. Surgery for primary intracerebral hemorrhage: is it safe and effective? Stroke 1997;28:2126-2132.

21. Morgenstern LB, Demchuk AM, Kim DH, Frankowski RF, Grotta JC 
Rebleeding leads to poor outcome in ultra-early craniotomy for intracerebral hemorrhage. Neurology 2001;56:1294-1299.

22. Steiner I, Gomori JM, Melamed E. The prognostic value of the CT scan in conservatively treated patients with intracerebral hematoma. Stroke 1984;15:279-282.

23. Juvela S. Risk factors for impaired outcome after spontaneous intracerebral hemorrhage. Arch Neurol 1995;52:1193-1200.

24. Massaro AR, Sacco RL, Mohr JP, et al. Clinical discriminators of lobar and deep hemorrhages: the Stroke Data Bank. Neurology 1991;41: 1881-1885.

25. Cheung RT, Zou LY. Use of the original, modified, or new intracerebral hemorrhage score to predict mortality and morbidity after intracerebral hemorrhage. Stroke 2003;34:1717-1722.

26. Becker KJ, Baxter AB, Cohen WA, et al. Withdrawal of support in intracerebral hemorrhage may lead to self-fulfilling prophecies. Neurology $2001 ; 56: 766-772$.

\section{Asymptomatic sinovenous thrombosis in a healthy neonate}

Meredith R. Golomb, MD, MSc; Mary Edwards-Brown, MD; and Bhuwan P. Garg, MBBS, Indianapolis, IN

Our patient's parents became concerned about a ridge on the back of his skull when he was 2 weeks old. He was otherwise healthy. Head CT demonstrated a normal skull but suggested sinovenous thrombosis; follow-up MRI and venography confirmed this (figure). He showed no signs of dehydration or seizures. His neurologic exam was normal. Prothrombotic evaluation was unremarkable. He was followed conservatively, with repeat imaging and neurologic exams. During the next year, follow-up CT venography revealed gradual resolution of thrombosis. He had normal head growth and development and a normal neurologic exam at 1 year of age.

Copyright @ 2006 by AAN Enterprises, Inc.

Dr. Golomb is supported by the following grants: NIH NINDS K23 NS 048024 and Clarian Values Fund grant \# VFR-171.

Disclosure: The authors report no conflicts of interest.

Address correspondence and reprint requests to Dr. Meredith R. Golomb, Indiana University School of Medicine, Building XE-040, 575 West Drive, Indianapolis, IN 46202; e-mail: mgolomb@iupui.edu

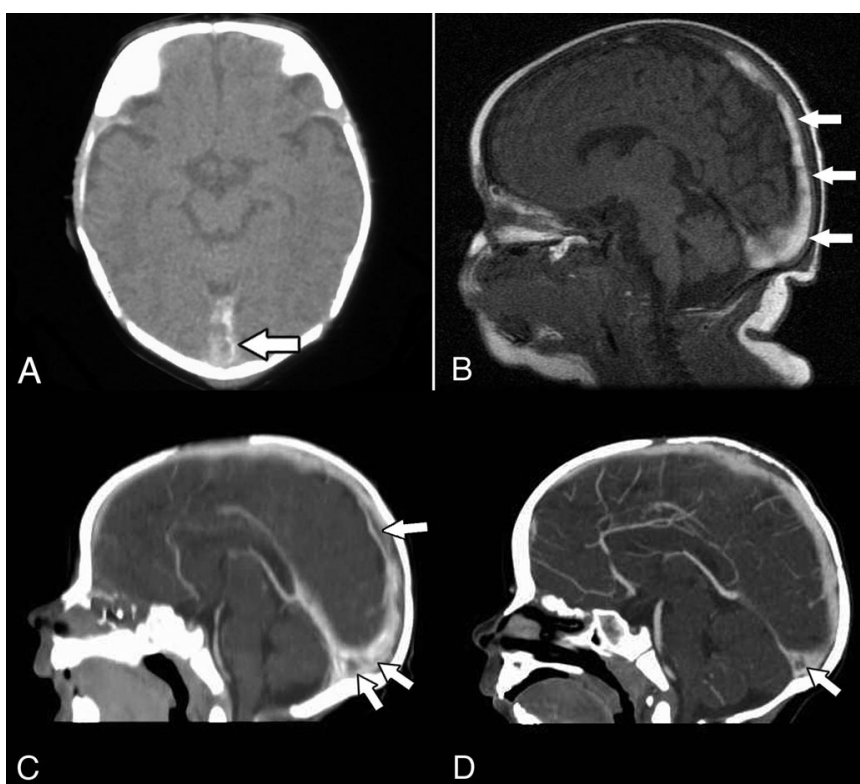

Figure. (A) Head CT at 15 days old: enlarged straight sinus. (B) T1-weighted MRI at 17 days old: extensive thrombus in sagittal sinus. (C) CT venography (CTV) at 2 months old: residual thrombus at the torcula and sagittal sinus. (D) CTV at 10 months old: minimal residual thrombus at the torcula. 


\title{
Neurology
}

\author{
Asymptomatic sinovenous thrombosis in a healthy neonate \\ Meredith R. Golomb, Mary Edwards-Brown and Bhuwan P. Garg \\ Neurology 2006;66;1186 \\ DOI 10.1212/01.wnl.0000195277.90853.bf
}

This information is current as of April 24, 2006

Updated Information \& Services

Citations

Permissions \& Licensing

Reprints including high resolution figures, can be found at: http://n.neurology.org/content/66/8/1186.full

This article has been cited by 1 HighWire-hosted articles: http://n.neurology.org/content/66/8/1186.full\#\#otherarticles

Information about reproducing this article in parts (figures,tables) or in its entirety can be found online at:

http://www.neurology.org/about/about_the_journal\#permissions

Information about ordering reprints can be found online: http://n.neurology.org/subscribers/advertise

Neurology ${ }^{\circledR}$ is the official journal of the American Academy of Neurology. Published continuously since 1951, it is now a weekly with 48 issues per year. Copyright . All rights reserved. Print ISSN: 0028-3878. Online ISSN: 1526-632X.

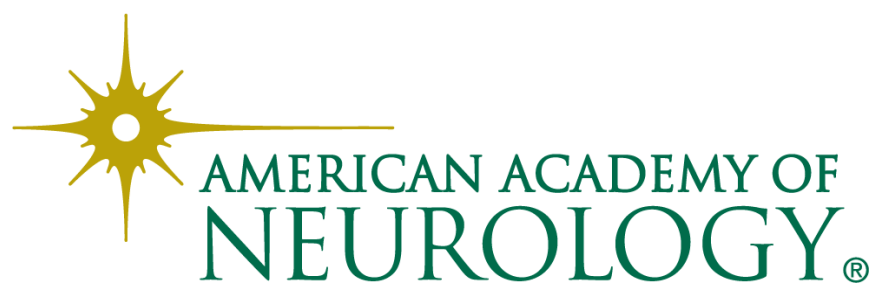

